TAO, Vol. 15, No. 4, 743-756, November 2004

NOTES AND CORRESPONDENCE

\title{
An Improved Estimate of Layer Thickness in the Atmosphere Using Dual-Frequency Interferometry Method of MST-VHF Radar
}

\author{
Jenn-Shyong Chen ${ }^{1, *}$ and Yen-Hsyang $\mathrm{Chu}^{2}$ \\ (Manuscript received 17 June 2003, in final form 13 August 2004)
}

\begin{abstract}
The purpose of this study was to obtain a more precise estimate of the thickness of an atmospheric layer structure, based on the theoretical works of Chen and Chu (2001), using a modified dual-frequency interferometry method of MST radar. Horizontal layer structures in the atmosphere are frequently formed due to vertically confined refractivity irregularities. The thickness and position of a layer structure can be measured by Frequency Domain Interferometry (FDI) technique, using two or more carrier frequencies. The condition of a single-Gaussian layer is generally assumed to facilitate the mathematical manipulation in the FDI method but this rigorous condition is very difficult to satisfy due to multiple layers and varied atmospheric structures. Consequently, the thickness and position of a layer, obtained by the FDI method, may differ from true ones. Chen and Chu (2001) verified this, in results obtained from their theoretical/numerical examination. Moreover, they found various kinds of relationships between the thickness and position of a layer. These findings have been attributed to the coupling of various layer structures and the range weighting function of the radar system. The thickness-position relationship indicates that the estimated layer thickness might be closer to the true one at some places more than others, depending on the layer structure involved in the radar volume. This study examined dual-frequency observations carried out with the Chung-Li VHF radar, to reveal the prevailing thickness-position relationship and deduce the potential layer structure. In general, a thicknessposition relationship with a smaller thickness at the central height of the
\end{abstract}

\footnotetext{
${ }^{1}$ Department of Electronic Engineering, Chienkuo Technology University, Changhua, Taiwan, ROC

2 Institute of Space Science, National Central University, Chung-Li, Taiwan, ROC

* Corresponding author address: Prof. Jenn-Shyong Chen, Department of Electronic Engineering, Chienkuo Technology University, Changhua, Taiwan, ROC; E-mail: jschen@ctu.edu.tw
} 
radar volume was obtained. This result was possibly due to multiple layers (more than two layers) or a single layer in company with significant background scatterers. According to numerical calculations, this kind of thickness-position relationship indicates that the thickness at the central height of the radar volume is closer to the true one. A quasi-linear relationship between layer thickness and radar pulse length was also observed, which demonstrated further that multiple layers and background scatterers have a significant impact on the thickness-position relationship of a layer. Since the radar volume for a short pulse length might contain just a single layer and fewer background scatterers, the data of 1- $\mu$ s pulse length were adopted to estimate approximate layer thickness. Using this approximation and taking into account the thickness around the central height of the sampling gate, the estimated layer thicknesses were found to be around $\mathbf{3 0}$ meters and approximately $70 \%$ of them were smaller than 60 meters.

(Key words: Atmospheric layer structure, Chung-Li VHF radar, Frequency domain interferometry)

\section{INTRODUCTION}

Atmospheric refractive index is related to atmospheric pressure, temperature, humidity, and electron density and can change remarkably in vertical direction and horizontal direction. Vertical changes have been demonstrated in a number of in-situ measurements with highresolution radiosondes. These measurements indicate that with sharp vertical gradients of temperature/humidity, multiple thin sheets, some as thin as a few decimeters, occur frequently in the troposphere and stratosphere (Dalaudier et al. 1994; Luce et al. 1995; Muschinski and Wode 1998). Spatial/temporal variations in refractive index also result in so-called, "atmospheric irregularities". These irregularities are often confined vertically but also extended horizontally, leading to the formation of a layer structure. In fact, many observations made by Continuous-Wave $(\mathrm{CW})$ radars, with very fine range resolutions such as 1,2 , and $20 \mathrm{~m}$, have revealed that thin echo layers or fine structures with a thickness from one meter to tens of meters exist in the planetary boundary layer and lower stratosphere (e.g., Richter 1969; Eaton et al. 1995; Cho et al. 1996). Observations made by pulsed radars, such as MST-VHF radar and UHF radar, have also frequently revealed echo layers. However, the finite range resolution of the pulsed radar makes it difficult to identify the multiple thin layers embedded in the radar volume even though the range resolution is as narrow as $75 \mathrm{~m}$ (Rüster 1997, 1998).

The MST radar community employs a technique of transmitting two or more frequencies to measure the thickness and position of a layer structure in the radar volume. This mode of measure is known as the Frequency Domain Interferometry (FDI) technique. The initial FDI technique used two frequencies and assumed the condition of a single-Gaussian layer in the radar volume to facilitate mathematical manipulation (Kudeki and Stitt 1987). Since multiple layers were thought to be usually responsible for radar returns (Luce et al. 1999; Palmer et al. 
1999; Chen and Chu 2001), an advanced FDI technique, using more transmitting frequencies, was introduced (Palmer et al. 1999). The numerical calculations of Chen and Chu (CC) (2001) have also demonstrated that when dual frequencies are employed, background scatterers and a non-Gaussian layer could cause incorrect estimates of layer thickness and position. In this study, however, the advanced FDI technique was not employed. Instead, a modified application of the conventional dual-frequency FDI method was proposed to obtain a more precise estimate of the layer thickness, based on the theoretical works of $C C$.

This paper is organized as follows. In section 2, we describe the theoretical/numerical results obtained by $C C$ and introduce the dual-frequency FDI method briefly. Observations, from the Chung-Li VHF radar with different pulse lengths, are presented in section 3. In section 4, potential layer structures for the observations are discussed. Finally, section 5 offers brief conclusions.

\section{FDI METHOD AND PREVIOUS THEORETICAL RESULTS}

\subsection{FDI Method}

Using dual-frequency FDI method, a cross-correlation function of two radar returns is defined by the following calculation:

$$
\mathrm{S}_{12}=\frac{<V_{1} V_{2}^{*}>}{<\left|V_{1}\right|^{2}>^{1 / 2}<\left|V_{2}\right|^{2}>^{1 / 2}}=\left|\mathrm{S}_{12}\right| \exp (j \phi),
$$

where $V_{1}$ and $V_{2}$ are the two radar returns, with transmitting frequencies $f_{1}$ and $f_{2}$, respectively. The asterisk stands for complex conjugate, angle brackets mean ensemble average over a period of time, $\left|S_{12}\right|$ and $\phi$ are magnitude and phase, respectively. According to (1) and under the assumption of a single layer with Gaussian distribution in the radar volume, several FDI analytical expressions were derived that can be employed to estimate layer thickness and layer position (Kudeki and Stitt 1987; Franke 1990; Liu and Pan 1993; Chu and Chen 1995; Chen et al. 1997). Although various FDI analytical expressions result in different values of thickness and position of the layer, they do not lead to diverse conclusions for this study. Therefore, only the thickness and position of the layer estimated with the expressions given by Chen et al. (1997) are shown in this article. The FDI equations used are as follows:

$$
\mathrm{S}_{12}=\frac{\left[\frac{1}{\sigma_{t}^{2}}+\left(\frac{4 k_{1}^{2}}{z_{0}^{2}}+\frac{1}{\sigma_{t}^{4}}\right) \frac{l_{t}^{2}}{4}\right]^{1 / 2}\left[\frac{1}{\sigma_{t}^{2}}+\left(\frac{4 k_{2}^{2}}{z_{0}^{2}}+\frac{1}{\sigma_{t}^{4}}\right) \frac{l_{t}^{2}}{4}\right]^{1 / 2}}{\left\{\left\{\frac{1}{\sigma_{t}^{2}}+\left[\frac{\left(k_{1}+k_{2}\right)^{2}}{z_{0}^{2}}+\frac{1}{\sigma_{t}^{4}}-\frac{\Delta k^{2}}{z_{0}^{2}}\right] \frac{l_{t}^{2}}{4}\right\}^{2}+\left(\frac{\Delta k}{z_{0}}+\frac{\Delta k l_{t}^{2}}{2 \sigma_{t}^{2} z_{0}}\right)^{2}\right\}^{1 / 2}}
$$




$$
\begin{aligned}
& \cdot \exp \left(-4 \sigma_{r}^{4} \Delta k^{2} / D_{z}^{2}\right) \exp \left[j\left(-2 z_{0}+4 \sigma_{r}^{2} z_{l} / \sigma_{l}^{2}\right) \Delta k\right] \exp (-j \theta), \\
& D_{z}^{2}=2 \sigma_{r}^{2}+l_{z}^{2} / 4, \\
& 1 / \sigma_{r}^{2}=1 / \sigma_{z}^{2}+2 / \sigma_{l}^{2},
\end{aligned}
$$$$
\theta=\tan ^{-1}\left\{\frac{\frac{\Delta k}{z_{0}}\left(1+\frac{l_{t}^{2}}{2 \sigma_{t}^{2}}\right)}{\frac{1}{\sigma_{t}^{2}}+\left[\frac{\left(k_{1}+k_{2}\right)^{2}}{z_{0}^{2}}+\frac{1}{\sigma_{t}^{4}}-\frac{\Delta k^{2}}{z_{0}^{2}}\right] \frac{l_{t}^{2}}{4}}\right\},
$$

where $k_{1}$ and $k_{2}$ are the wave numbers corresponding to the transmitting frequencies $f_{1}$ and $f_{2}, \Delta k=k_{2}-k_{1}, z_{0}$ is the vertical coordinate of the radar receiver with respect to the center of the radar volume, $\sigma_{t}=2^{1 / 2} h \theta_{6} / 3.33, \theta_{6}$ is the 6 - $\mathrm{dB}$ angular width of the two-way antenna pattern function, $h$ is the central height of the radar volume, $\sigma_{z}=0.35 c \tau / 2, c$ is the speed of light and $\tau$ is the pulse length, $z_{l}$ is the position of the layer with respect to the central height of the radar volume, and $\sigma_{l}$ is the thickness of the layer (the second moment of a Gaussian function). $l_{z}$ and $l_{t}$ are, respectively, the spatial correlation lengths of the refractive irregularities in vertical and horizontal directions. Note: Eg. (2) is used only for the observation with vertical radar beam.

Since Eg. (2) is only valid for the condition of a single-Gaussian layer, the estimated layer position and thickness could be quite different from the true ones if the layer is not Gaussian shape or multiple layers exist in the radar volume. In the following some numerical results presented by $C C$ are introduced briefly.

\subsection{Previous Theoretical Results}

$C C$ revealed a variety of relationships between layer thickness and layer position, using the exiting dual-frequency equations [e.g., Eq. (2)]. $C C$ also used theoretical/numerical examination to obtain thickness-position relationships similar to the ones observed, when considering the conditions of multiple layers, background scatterers, and non-Gaussian layer in the radar volume. Figure 1 shows some typical numerical results obtained by $C C$, where three types of thickness-position relationships (TPR) are demonstrated. The first TPR is demonstrated by minimum thickness at the central height of the radar volume, opening on right side; the second TPR is demonstrated by maximum thickness at the central height of the volume, opening on left side, and the third one is a quasi-linear TPR with a negative slope (A positive slope can also demonstrate a TPR.). In this article, these three patterns of TPR are referred to as Relations 1,2, and 3 respectively. $C C$ demonstrated that Relations 1, $2 \& 3$ could arise from the combined effects of non-uniform range weighting function and various layer structures moving up and down in the radar volume. The scenarios are summarized as follows:

- In Fig. 1a (left panel), that Relation 1 was obtained from a single layer with a smooth shape compared to that of a Gaussian layer. 

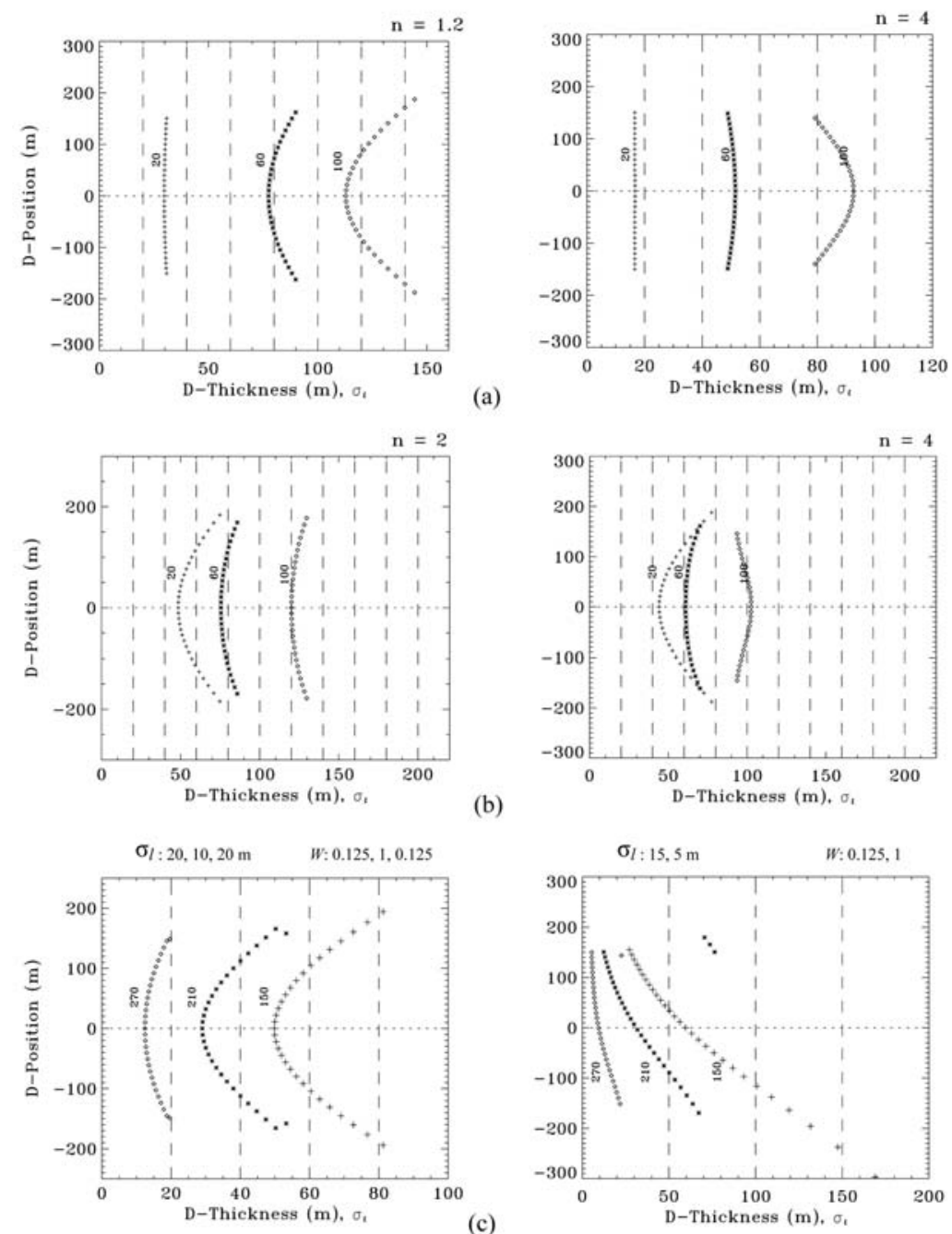

Fig. 1. Typical thickness-position relationships obtained by Chen and Chu (2001). (a) A single layer with the shape $\exp \left(-\left|z-z_{l}\right|^{\mathrm{n}} / 2 \sigma_{l}^{\mathrm{n}}\right), \mathrm{n}=1.2$ and 4 for the left and right panels. $z_{l}$ and $\sigma_{l}$ are, respectively, the position and thickness of the layer. The numbers attached on different symbols are the given thicknesses (in unit of meter). (b) Similar to (a) but in company with background scatterers. (c) left panel: three-layer structure, right panel: two-layer structure. The values of $\sigma_{l}$ are the thicknesses of the upper, middle, and lower layers (left panel) or the upper and lower layer (right panel). The parameter of $W$ indicates the contribution weight of the layer. The numbers attached on different symbols are the distances (in unit of meter) between the central and adjacent layers (left panel) or between the two layers (right panel). 
- In Fig. 1b (left panel), that Relation 1 was also obtained from a single-Gaussian layer with background scatterers.

- In Fig. 1a (right panel), that Relation 2 was obtained from a non-Gaussian layer, with sharper boundaries than that of a Gaussian layer.

- In Fig. $1 \mathrm{~b}$ (right panel), with a layer thickness of $100 \mathrm{~m}$, that Relation 2 was obtained in the case of a sharp layer with very large thickness, for that the role of background scatterers diminished.

- Relations 1, 2 and 3 were obtained when multiple layers, with different thicknesses, separations, and weights were moving in the radar volume. Relations 1 and 3 can be seen in the left panel and right panel of Fig. 1c, respectively. Note: Relation 3 is mainly generated from a two-layer structure.

Figure 1 clearly demonstrates that the layer thicknesses derived from the dual-frequency FDI equations would be different to the ones given in the model, when the condition of a single-Gaussian layer is not valid. Nonetheless, Figure 1 also demonstrates that the derived thicknesses are closer to the true ones when (1) the derived layers locate at around the zero level (the central height of the radar volume) for Relations 1 and 2, and (2) the layers locate near the upper (or lower) boundary of the gate for Relation 3. These features indicate that the dual-frequency method can be used to acquire a more precise estimate of layer thickness, as long as the estimated thickness-position relationship is obtained.

\section{RADAR OBSERVATION}

\subsection{Experimental Setup}

The Chung-Li VHF radar with vertical beam was used to collect FDI data, from 25 November 2000 1535LT to 27 November 2000 1035LT, and matched filter was used in receiving. Three radar pulse lengths, 4, 2 and $1 \mathrm{~s}$, were transmitted alternately, and the sampling intervals were 4, 2 and $1 \mu \mathrm{s}$, respectively. The dwell time, of each radar pulse operation in one cycle, was about one minute and the two carrier frequencies were 51.9 and $52.15 \mathrm{MHz}$, respectively. The time resolution of the raw data was $0.11 \mathrm{~s}$, and about one-minute of raw data was taken to obtain an FDI cross-correlation value in accordance with (1). The thickness and position of the layer were then estimated in terms of (2), irrespective of the true layer structure in the radar volume.

\subsection{Observation}

Figure 2 shows the scatter diagrams of layer position versus layer thickness estimated from the above data set for the height interval between about 3 and $5 \mathrm{~km}$, in which each scatter diagram was obtained from three-hour data. Here, the scatter patterns of the estimated position and thickness seemed to follow the TPR of Relation 1, as depicted in Fig.1. Although not shown here, most of the TPRs at other heights were also classified as Relation 1. It should be noted, that the three pulse lengths were transmitted alternately in the experiment and therefore the same atmospheric conditions were responsible for radar returns with different pulse lengths=. 
(a)

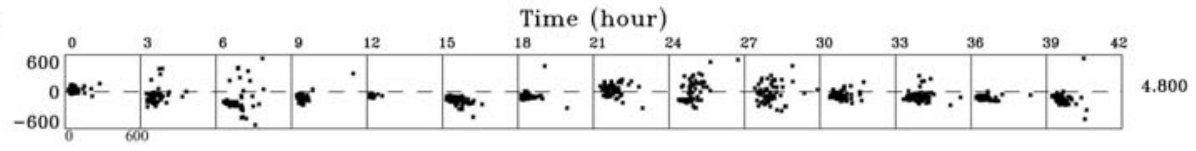

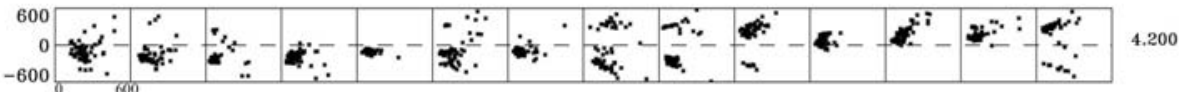

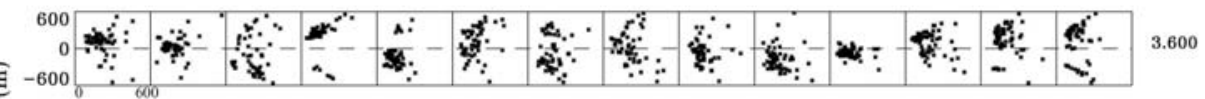

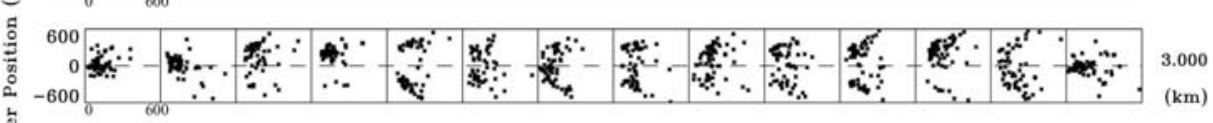
Layer Thickness (m)

(b)

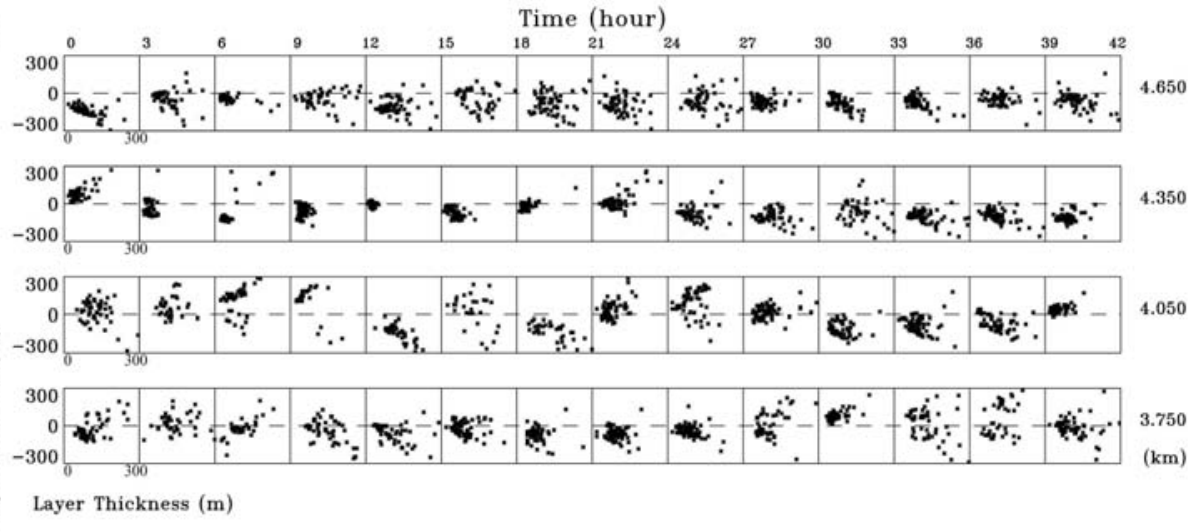

(c)

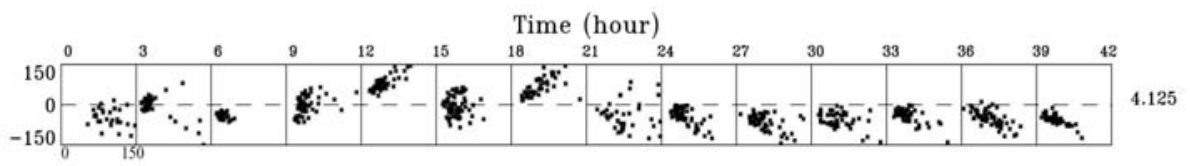

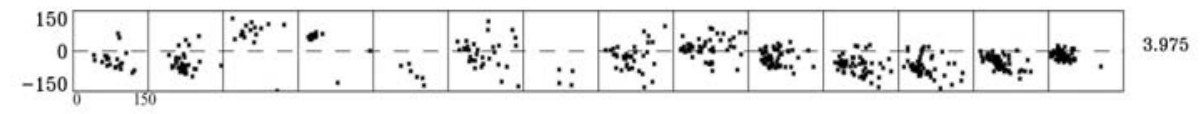

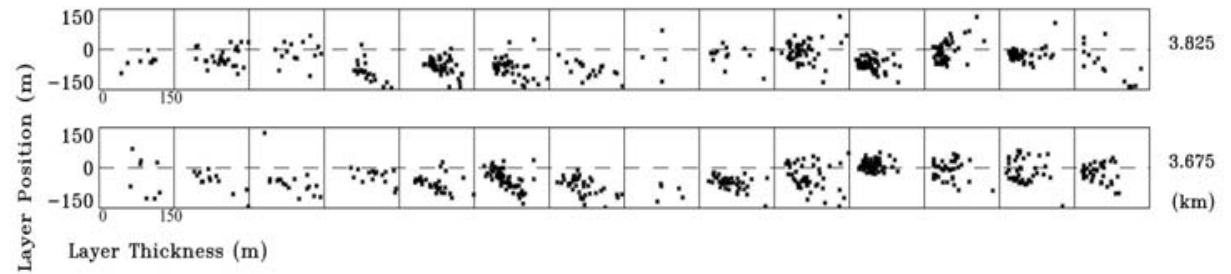

Fig. 2. Scatter diagram of layer position versus layer thickness for the observation conducted on 25-27 November 2000. (a), (b), and (c) present the data observed with 4-, 2-, and 1- $\mu$ s pulse lengths, respectively. Only the four range gates around the height of $4 \mathrm{~km}$ are shown. 
Figure 3 shows the averaged results of layer thickness versus layer position for the three sets of data depicted in Fig. 2, respectively. Apparently, these are also categorized as Relation 1 despite different mean values and standard deviations of layer thicknesses. In Fig. 3, the mean values of layer thicknesses at the position of zero are about 120,80 and $45 \mathrm{~m}$, respectively, for pulse lengths of 4,2 , and $1 \mu \mathrm{s}$. The standard deviations of layer thicknesses also vary with pulse length but are nearly independent of the layer position. On average, the layer thicknesses are about 75, 60, and $35 \mathrm{~m}$, respectively, for the three pulse lengths. The results depicted in Fig. 3 demonstrate a dependent relationship for the estimated layer thickness on radar pulse length.

Figure 4 shows the histograms of the estimated layer thicknesses for different pulse lengths. Evidently, both crest and breadth of the distribution are quasi-linear variation with radar pulse length. The probable causes responsible for the features displayed in Figs. 3 and 4 are discussed in the following section.

\section{DISCUSSION}

Dual-frequency FDI observation demonstrated that the estimated thicknesses for the layers situated at around the position of zero (the central height of the radar volume) were smaller than those located at places far from the radar volume center. Clearly, the atmospheric layer structure is not responsible for this behavior but rather the inadequate use of the exiting dualfrequency FDI equations to resolve complicate layer structures. From the numerical results obtained by $C C$, there are three typical patterns describing the thickness-position relationship (TPR) for different layer structures. In view of the various parameters of the layer structures,

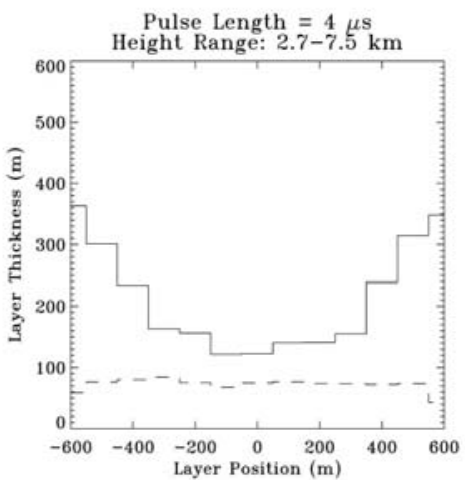

(a)

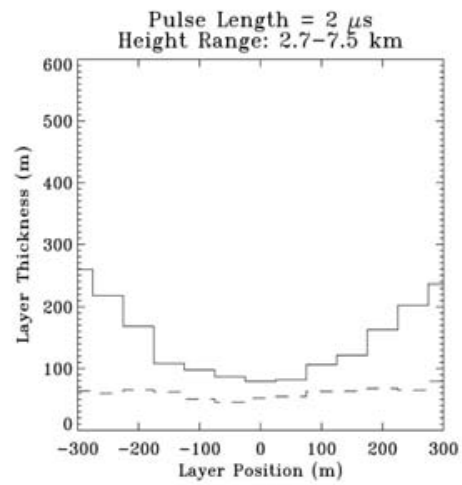

(b)

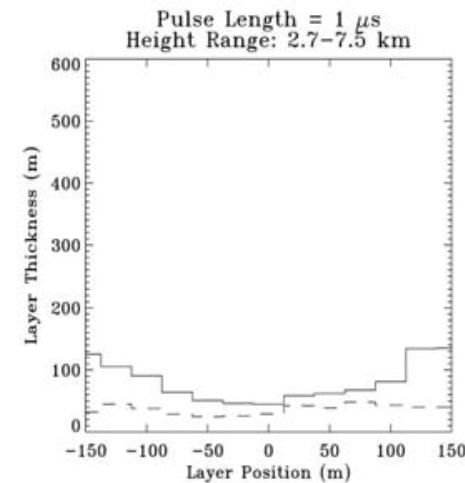

(c)

Fig. 3. Averaged layer thicknesses at different layer positions, where solid and dashed curves represent, respectively, mean value and standard deviation of layer thicknesses. (a), (b), and (c) demonstrate the data observed with 4-, 2-, and 1- $\mu$ s pulse lengths, respectively. 


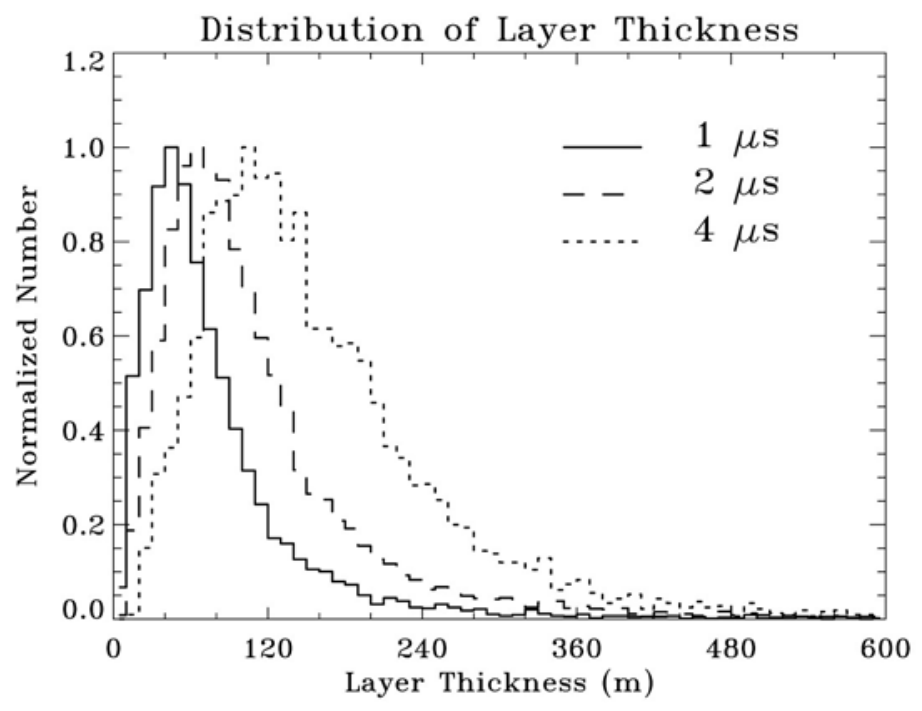

Fig. 4. Histograms of layer thicknesses for the data of 4-, 2-, and 1- $\mu$ s pulse lengths. Only those thicknesses located in the height interval of 2.7-7.5 $\mathrm{km}$ are adopted.

such as position, thickness, shape, contributing weight, separation, etc., the occurrences of different TPRs should have the same chance. Nonetheless, the prevailing TPR, in this study, was observed to be Relation 1. As $C C$ pointed out, the existence of background scatterers in the radar volume tends to favor Relation 1; therefore, the background scatterers might play an important role in the observation of TPR in this study. Other layer structures, such as multiple layers and those smoother than Gaussian layer, may also be responsible for the prevalence of Relation 1.

Both multiple layers and background scatterers are also able to explain the dependence of layer thickness on radar pulse length displayed in Figs. 3 and 4. Some studies have demonstrated that the layer thickness, estimated by the single-layer FDI equation, approximates to the separation distance of two thin layers in the radar volume (Luce et al. 1999; Palmer et al. 1999). For more than two layers, a numerical simulation can be performed as follows. Assuming that the layers have a thickness of $10 \mathrm{~m}$ and are equally spaced with a separation of $30 \mathrm{~m}$, the FDI coherence function can be estimated using the multiple-layer FDI equation given by $C C$ [see Eq. (7) in their paper or the Appendix in this paper]. A layer thickness is then estimated by substituting the resultant FDI coherence function into the equation of single-layer FDI equation, namely, Eq. (2). Figure 5 shows the results for two to five layers, in which the maximum separations between uppermost and lowest layers are 30,60,90, and $120 \mathrm{~m}$, for two, three, four, and five layers, respectively. Fig. 5 demonstrates that the estimated thickness is close to the maximum separation between the layers. Moreover, numerical computations obtained by considering the contribution of background scatterers revealed that the shorter the 
pulse length, the thinner the thickness would be (not present here). Since the numbers of layers/background scatterers in the radar volume for a short pulse length are generally fewer than those for a long pulse length, the numerical result depicted in Fig. 5 provides a potential interpretation of the quasi-linear dependent relationship between layer thickness and radar pulse length.

Since it is more likely for a short radar pulse to contain a single layer and a small amount of background scatterers, the layer thickness and position estimated from the data of 1- $\mu \mathrm{s}$ pulse length should be closer to the true ones, than those estimated from 2-and 4- $\mu$ s pulse lengths. In the case of multiple layers, the dominant layer would be located at around the central height of the radar volume due to its large weight from the range weighting effect. In view of these scenarios, a better estimate of layer thickness can be achieved by inspecting the data of $1-\mu$ s pulse length, with the estimated layer thickness at around the central height of the radar volume. Figure 6 shows the histogram of thicknesses for the data of $1-\mu$ s pulse length, where only those thicknesses located at a height interval between $-5 \mathrm{~m}$ and $5 \mathrm{~m}$, with respect to

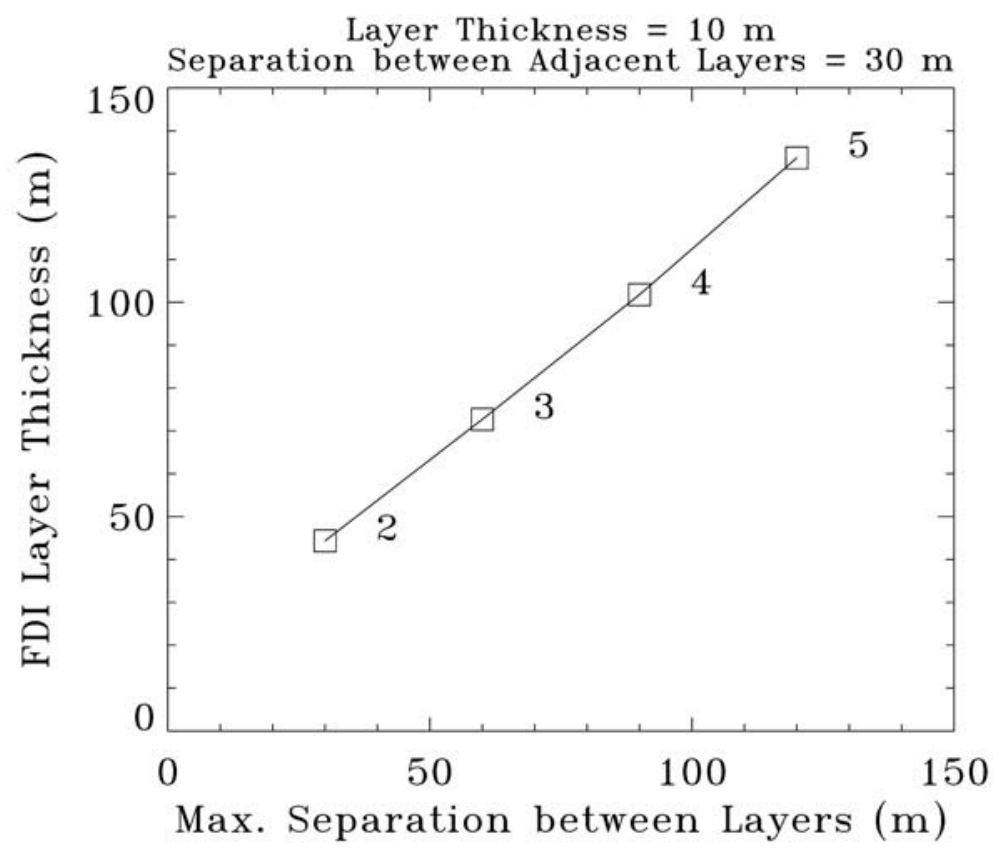

Fig. 5. Layer thickness (estimated with single-layer FDI expression) versus maximum separation between the multiple layers given in the numerical simulation. Two times the second moment of the Gaussian layer is referred to as the layer thickness in this plot. The digits attached to the square symbols are the numbers of layers given in the multiple-layer model. 
the radar volume center, were adopted in the histogram. Here, the distribution of thicknesses mean at around $30 \mathrm{~m}$, with about $70 \%$ of the thicknesses smaller than $60 \mathrm{~m}$. Since both multiple layer structures and background scatterers tend to broaden the estimated layer thickness, the actual layer would be expected to be often thinner than 30 meters.

The layer thicknesses presented in Fig. 6 are in agreement with those measured by highresolution CW radars; however, they seem to be generally higher than the thicknesses of the temperature/humidity sheets measured by high-resolution radiosondes.

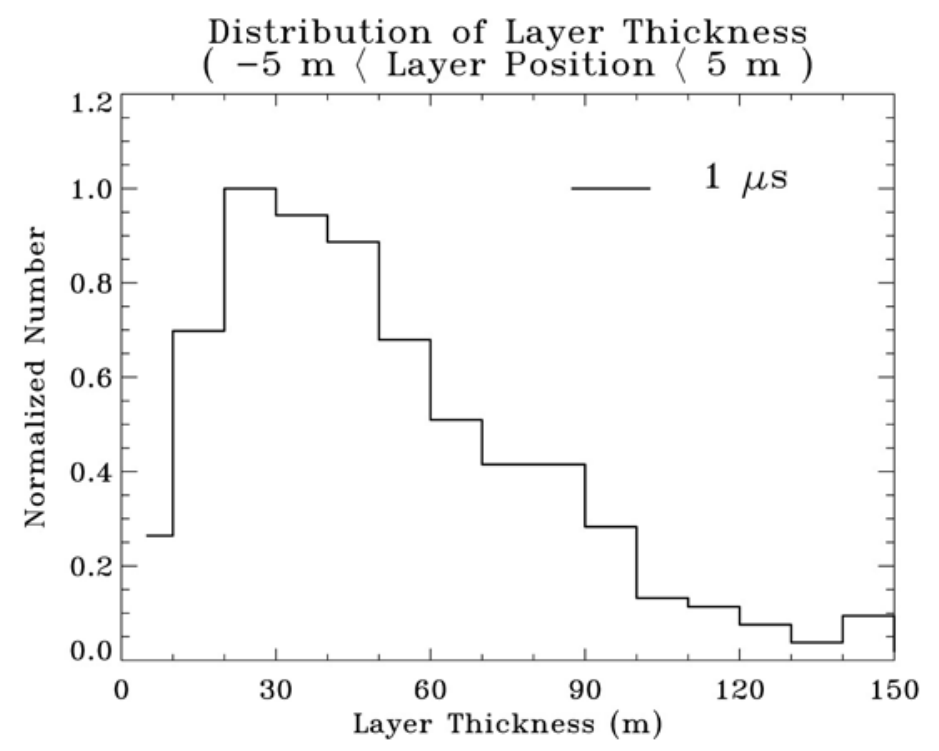

Fig. 6. Histogram of layer thicknesses for the data of $1-\mu$ s pulse length. Only those thicknesses located in the height interval of $-5 \mathrm{~m}$ and $5 \mathrm{~m}$, with respect to the radar volume center, are included.

\section{CONCLUSIONS}

In this paper, a modified application of the dual-frequency interferometry method is proposed to obtain a more precise measurement of layer thickness. The estimated layer thickness was found to be highly dependent on the estimated layer position in the radar volume, and the prevailing thickness-position relationship was characterized by a smaller thickness for the layer located at around the central height of the radar volume and a larger thickness for the layer situated farther from the radar volume center. According to the theoretical/numerical works of Chen and Chu (2001), a number of layer structures could result in this feature, including multiple layers (more than two) and/or a non-Gaussian layer in company with considerable background scatterers in the radar volume. For this thickness-position relationship, the 
estimated thickness near the central height of the radar volume is closer to the true one of the layer. Therefore, the data of 1- $\mu$ s pulse length, with the estimated thicknesses at around the central height of the radar volume, can be used to obtain a more precise estimate of layer thickness. This method revealed that the thicknesses of the layer structures were around $30 \mathrm{~m}$ and $\sim 70 \%$ of them were smaller than $60 \mathrm{~m}$. This magnitude of thickness is similar to that observed by $\mathrm{CW}$ radars, and close to that of the temperature/humidity sheets measured by high-resolution radiosondes.

Acknowledgements The National Central University, Taiwan maintains the Chung-Li VHF radar. The authors would like to thank C.H. Wu, engineer of the Chung-Li VHF radar, for assisting in the operation of the radar and the anonymous reviewers for their valued comments on this paper. The National Science Council under grants NSC91-2111-M-270-001, NSC922111-M-008-025, and NSC93-2111-M-270-001 have supported this work.

\section{REFERENCES}

Chen, J. S., J. Y. Liu, and Y. H. Chu, 1997: Effects of anisotropy and power spectrum of refractivity irregularities on determination of layer thickness and layer position using the frequency domain interferometry technique. Radio Sci., 32, 437-451.

Chen, J. S., and Y. H. Chu, 2001: Examination of the layer model of the frequency domain interferometry theory applied in mesosphere-stratosphere-troposphere radars.J. Atmos. Solar-Terr. Phys., 63, 235-251.

Cho, J. Y. N., R. F. Jurgers, and M. A. Slade, 1996: High-resolution stratospheric dynamics measurements with the NASA/JPL Goldstone Solar System Radar. Geophys. Res. Lett., 23, 1909-1912.

Chu, Y. H, and T. Y. Chen, 1995: Theoretical study of two-frequency coherence of MST radar returns. Radio Sci., 30, 1803-1815.

Dalaudier, F., C. Sidi, M. Crochet, and J. Vernin, 1994: Direct evidence of "sheets" in the atmospheric temperature field. J. Atmos. Sci., 51, 237-248.

Eaton, F. D., S. A., Mclaughlin, and J. R. Hines, 1995: A new frequency-modulated continuous wave radar for studying planetary boundary layer morphology. Radio Sci., 30, 7588.

Franke, S. J., 1990: Pulse compression and frequency domain interferometry with a frequencyhoped MST radar. Radio Sci., 25, 565-574.

Kudeki, E., and G. R. Stitt, 1987: Frequency domain interferometry: A high-resolution radar technique for studies of atmospheric turbulence. Geophys. Res. Lett., 14, 198-201.

Liu, C. H., and C. J. Pan, 1993: New observational techniques for studying the dynamics of the middle atmosphere using the Chung-Li VHF radar.J. Atmos. Terr. Phys., 55, 10551066.

Luce, H., M. Crochet, F. Dalaudier, and C. Sidi, 1995: Interpretation of VHF ST radar vertical echoes from in situ temperature sheet observations. Radio Sci., 30, 1003-1025. 
Luce, H., M. Crochet, C. Hanuise, M. Yamamoto, and S. Fukao, 1999: On the interpretation of the layered structures detected by mesosphere-stratosphere-troposphere radars in dual frequency domain interferometry mode. Radio Sci., 34, 1077-1083.

Muschinski, A., and C. Wode, 1998: First in situ evidence for coexisting submeter temperature and humidity sheets in the lower free troposphere. J. Atmos. Sci., 55, 2893-2906.

Palmer, R. D., T. Y. Yu, and P. B. Chilson, 1999: Range imaging using frequency diversity. Radio Sci., 34, 1485-1496.

Richter, J. H., 1996: High resolution tropospheric radar sounding. Radio Sci., 4, 1261-1268.

Rüster, R., 1997: High resolution measurements in the summer polar mesosphere. Geophys. Res. Lett., 24, 1115-1118.

Rüster, R., G. D. Nastrom, and G. Schmidt, 1998: High-resolution VHF radar measurements in the troposphere with a vertically pointing beam. J. Appl. Meteor., 37, 1522-1529.

\section{Appendix: Dual-frequency FDI equation for multiple layers}

Assuming the radar echoes are scattered from N Gaussian layers in the radar volume, the cross-correlation function for two radar echoes with different transmitting frequencies can be derived as [see Chen and Chu (2001) for detailed derivation]

$$
\left\langle V_{1} V_{2}^{*}\right\rangle=\frac{\sqrt{2} C^{2} \pi^{3}}{z_{0}^{4} \sqrt{a_{x} a_{y} A_{x} A_{y}}} \sum_{n=1}^{\mathrm{N}} \frac{w_{n}^{2} \sigma_{r_{n}} \mathrm{e}^{-z_{l_{n}}^{2} / \sigma_{l_{n}}^{2}} \mathrm{e}^{-\left(2 k_{1}+\Delta k\right)^{2} / 4 A_{z_{n}}} \mathrm{e}^{-2 \sigma_{r_{n}}^{2} \Delta k^{2}} \mathrm{e}^{2 \sigma_{r_{n}}^{2} z_{l_{n}}^{2} / \sigma_{l_{n}}^{4}} \mathrm{e}^{j\left(-2 \Delta k z_{0}+4 \sigma_{r_{n}}^{2} \Delta k z_{l_{n}} / \sigma_{l_{n}}^{2}\right)}}{\sqrt{A_{z_{n}}}},
$$

where $C$ is a constant related to the radar parameters, $\mathrm{z}_{0}$ is the central height of the radar volume, $w_{n}$ is the weight of the $n^{\text {th }}$ layer and related to the intensities of the refractive index fluctuations in the layer, $k_{1}$ is the wavenumber of the first transmitting frequency and $k_{2}$ for the second transmitting frequency, $\Delta k=k_{2}-k_{1}, z_{l n}$ and $\sigma_{l n}$ represent the position and thickness of the $n^{\text {th }}$ layer, respectively, and

$$
\begin{aligned}
& 1 / \sigma_{r_{n}}^{2}=1 / \sigma_{z}^{2}+2 / \sigma_{l_{n}}^{2}, \\
& a_{x}=1 / \sigma_{x}^{2}+j \Delta k / z_{0}, \\
& a_{y}=1 / \sigma_{y}^{2}+j \Delta k / z_{0}, \\
& b=\left(2 k_{1}+\Delta k\right)^{2} / z_{0}^{2}, \\
& A_{x}=\left(b / a_{x}+a_{x}+4 / \alpha l_{x}^{B}\right) / 4, \\
& A_{y}=\left(b / a_{y}+a_{y}+4 / \alpha l_{y}^{B}\right) / 4, \\
& A_{z_{n}}=1 / 8 \sigma_{r_{n}}^{2}+1 / \alpha l_{z}^{B},
\end{aligned}
$$


where $\sigma_{z}=0.35 c \tau / 2, c$ is the light speed and $\tau$ is the pulse length, $\sigma_{x}$ and $\sigma_{y}$ are, respectively, the second moments of the radar beam in $x$ and $y$ directions, $l_{x}, l_{y}$, and $l_{z}$ are, respectively, the spatial correlation lengths of the refractive irregularities in $x, y$, and $z$ directions, $\alpha$ and $\beta$ are the coefficients related to the wavenumber power spectrum of refractive irregularities. Note: $\alpha=1$ and $\beta=2$ for the 3-dimensional Gaussian spectrum, and $\alpha=2.5 l_{z}^{-1.4}$ and $\beta=1.5$ for the approximation form of the 3 -dimensional $-11 / 3$ power law spectrum. For the details, refer to Chen et al. (1997). To obtain the FDI coherence function, (Eg. A1) has to be normalized by $\left.<\left|V_{1}\right|^{2}\right\rangle^{1 / 2}<\left|V_{2}\right|^{2}>^{1 / 2}$, where $\left|V_{1}\right|^{2}$ can be obtained from (Eg. A1) by setting $\Delta k=0$, and $\left|V_{2}\right|^{2}$ is acquired by setting $\Delta k=0$ and replacing $k_{1}$ by $k_{2}$. 